(carbolic acid) and glycerin. It seems to work better. Considerable experimental work has been dorse on the effect of draughts and cold on infected ears. Dr. J. J. Moore and I found that rabbits that were exposed to the electric light rays in a closed box under a proper regulated temperature controlled by a thermostat either did not develop lesions or had fewer or less severe lesions than those that were not so treated. I found that children would readily get otitis media when exposed to drafts after colds or grippal infections. I attended three families in succession in the same flat, which was cold and damp. I had a child with acute otitis media and told the parents that I did not think we would get good results from treatment unless we removed the child to a hospital. The child was taken to a hospital, and the first night the symptoms subsided, the temperature went down, and the pain disappeared. After about ten days, the child was returned to the flat and the next day there was a complete recurrence of pain and symptoms. I apply heat to the part by using an electric bulb, hot boric acid applications or a hot water bottle. A hot salt bag is very useful. Any one can apply the rays from an electric light bulb continuously to the region of the ear.

Dr, Oliver W. Hill, Knoxville, Tenn.: I would urge a routine examination of every child that comes under observation if it shows any sign of coryza or upper respiratory tract infection. Many otologists are not certain as to when to interfere with an acutely infected ear and when to practice watchful waiting. I should like to hear some one who has had experience explain how to determine, by the physical appearance of the ear, the type to interfere with and the type to let alone.

Dr. Henry Dietrich, Los Angeles: As to the time to interfere with ears, I do not believe that the physical appearance of the ear drum alone can determine that. Another factor is that the virulence of the infection varies from year to year. An ear which must be incised during one epidemic need not be incised during another. During the epidemic two years ago, It was found advisable to incise almost every ear that showed any particular change, and at that time complications on the part of the mastoid and sinuses were common. This year our experience is quite different; that is, an ear drum which had the appearance of the ear drum of two years ago would heal under proper medical care.

Dr. Clifford D. Sweet, Oakland, Calif.: Too much emphasis cannot be placed on the routine examination of the child's ear. It is important to impress on parents and general practitioners the importance of this as part of the general examination, and that there is no symptom on which you can depend to make the diagnosis. I have seen the most virulent infection, with no sign from the child that would call attention to the ear. One must be able to recognize the normal drum and also the appearance of the drum as the inflammatory process recedes or progresses. With regard to the question as to whether to incise or to wait: First, if there is any doubt, a skilfully done paracentesis can do no harm; but it should be remembered that if the paracentesis is made too early before the process in the ear has progressed sufficiently, the incision will close up in from twenty-four to thirtysix hours, only the upper part remaining open, and you will have to repeat the paracentesis. Of course, all of the clinical symptoms of the condition must be taken into consideration in deciding when to interfere and when not to interfere. The question as to when to interfere in mastoid involvement requires more skilful team work than any other single point. No ear discharges very long without involvement of the mastoid, and I think that as soon as it can be definitely decided that the mastoid is involved, it should be interfered with surgically because of the serious consequences which may follow. I was on the advisory board through which men passed in the draft, particularly from such communities as San Francisco Bay, where the climate is damp, and 4 per cent. of the men were rejected because of chronic ear conditions. We found that they had chronic ear disease which lighted up with colds. It was apparent that these cases could have been cleared up by operations on the ear or tonsils and adenoids at the proper time.

Dr. W. McKim Marriotr, St. Louis : I would like to draw attention to two points in the symptomatology of otitis media in infants. One is that the otitis media may occur without any rise in temperature, even when there are definite signs of pus in the middle ear. The only symptom noted may be a failure to gain in weight. The second important symptom which may also occur, even in the absence of fever, is vomiting. This symptom is often promptly relieved following paracentesis.

Dr. S. Herman Lippitr, Milwaukee: Something was said about the child's holding the hands to the ear or rolling the head from side to side in middle ear disease. I have found those symptoms absolutely untrustworthy. The only way to make a diagnosis is by the routine use of the electric otoscope. I have found that following cases of tonsillitis and upper respiratory infections, many cases of ot itis media developed one year, and the next year, following the same types of infections, cases of pyelitis were numerous.

Dr. A. Spencer Kaufman, Philadelphia: With regard to the indications for incision, there is one point to which I want to call attention, and that is, a retraction of the membrane with redness in an acute catarrhal otitis media is really a contraindication to incision; and, when a membrane is incised too early, the slight hissing sound is caused by the air rushing into the vacuum that has been created by the sealing off of the tube. I was glad to hear Dr. Dietrich mention the varieties of infection one meets in different years. This year it was not an uncommon thing to see a septic temperature (such as one would expect to find in a sinus thrombosis) accompanying a simple otitis media which cleared up without a surgical mastoid involvement. The experience in examining the draft men very forcibly brings to our attention the necessity of the early care of these cases. There is no excuse for a chronic discharging ear. If the discharging persists, postauricular drainage will, in practically all cases, clear it up. I think the class of cases that Dr. Marriott referred to, the afebrile cases of otitis with discharge, usually comes during the period of dentition in infancy. We all must recognize that infection of the middle ear comes too frequently during dentition to be a mere coincidence, and very often the first symptom will be the evidence of discharge.

\section{SEQUELAE OF EPIDEMIC (LETHARGIC) ENCEPHALITIS *}

WILLIAM HOUSE, M.D. PORTLAND, ORE.

Recent literature on epidemic encephalitis has been so extensive that any addition may be a bore. Nevertheless, such a probability is here risked in the hope of presenting a reasonably simple working classification of the sequelae of that disease. H. G. Wells, writing of the education of the middle part of the last century, recently said, "The test of a good style was its abundance of quotations, allusions and stereoptyped expressions." With all deference to the extensively reviewed writing and opinions of others, the practice that Wells scorns will be avoided on the ground that the literature is readily available and that time limits will permit of few excerpts. 'These remarks are based on an examination of 145 patients with epidemic encephalitis, ninety of whom were examined during acute and fifty-five during chronic stages of the disease or because of sequelae. Many of the patients were examined at remote distances and under circumstances that prevented detailed records. Accordingly, an exact statistical study will not be attempted, nor is it essential for present purposes. Be it sufficient that no case was included that seemed diagnostically doubtful; but, conversely, that to the list might be added a considerable

* Read before the Section on Nervous and Mental Diseases at the Seventy-Third Annual Session of the American Medical Association, St. Louis, May, 1922 
number of cases insufficiently investigated which were probably due to epidemic encephalitis.

Of the ninety acute cases, twenty-four are known to have been fatal, giving a known mortality of 26 per cent., with six or seven cases that may have terminated and probably did terminate fatally, so that it is quite likely that the total mortality was nearer 33 per cent. The cases here discussed were taken from the remainder of these ninety cases plus the group of fifty-five cases seen during chronic stages or because of sequelae, which cases were themselves the residue of other groups whose mortality is unknown. All of these cases occurred between late October, 1919, and January, 1922. The epidemic which furnished them moved in waves, the first lasting during October, November and December, 1919, with lesser waves in Jantuary and March, and complete subsidence by May, 1920 . During this period, fifty-four acute cases were examined. From October, 1920, to April, 1921, there was an almost continuous series numbering thirty-six acute cases which were much less severe than those of the preceding winter, with a death rate little more than half as great.

The succeeding remarks will be limited to the subject matter predicated by the title of the paper.

\section{COMPLETE RECOVERY}

So many patients have been left with disagreeable sequelae that doubt has often been expressed as to whether any patient with epidemic encephalitis ever fully recovers. Apparently many have been left little the worse as a result of this infection. After deducting the fatal cases, probably one third of the patients are now well, though with rare exceptions convalescence was prolonged and he was indeed fortunate who, having suffered from encephalitis, was quite well at the end of a year, though many resumed work in from three to six months. The remainder are still uncomfortable, many of them helpless, more in a condition of semi-invalidism which permits of some activities but robs life of much of its joy. These unfortunates may be divided into four principal groups. For descriptive purposes, the order of grouping is that of increasing ratio of interest rather than of numbers.

\section{FOUR GROUPS}

Group 1: This group is least numerous, though not without interest, and is composed of those who in the acute stages exhibited myoclonus symptoms and who still suffer from muscular jerking and twitching, though in steadily decreasing severity. There are but two pronounced cases in the nonfatal group, and in each, stabbing, lightning, neuritic pains characterized the early stages of the disease, and the patients still have considerable twitching but without pain. They exhibit neurasthenic symptoms, have performed no continuous labor, and belong also in Group 3. They will not here be further discussed.

Group 2: This is composed of those who exhibited definite psychoses. The most common characteristics of these were suggestive of dementia praecox, with one paranoid case.

Group 3: This is composed of those who showed symptoms suggestive of neurasthenia or psychoneurasthenia. No attempt will here be offered to theorize on the pathologic reasons for the persistence of symptoms as between organic lesion, toxic condition, or pure psychopathy. This is the largest of the four groups.

Group 4: This group is composed of those who exhibited signs of gross organic lesion of the brain or cord. It may be divided into three subgroups, the symptoms of which suggest :

(a) A single unilateral brain lesion producing some such disorder as hemiparesis.

(b) Lesions limited to the pons or medulla or both,, causing persistent oculomotor or bulbar disturbances.

(c) Disseminated lesions, most classically revealed in the production of parkinsonian symptoms with many attendant and variable side effects.

\section{PSYCHOSES}

Psychoses (Group 2) were, of course, present in a large number of the acute cases. They persisted or developed as sequelae in not less than five cases, but had no pathognomonic characteristics. The symptoms suggested that the patients were predisposed to mental disorders and that the psychoses were excited rather than caused by the infection. Four of these patients were constitutional psychopathic inferiors, and in each the manifestations were typical of dementia praecox with plainly discernible hereditary elements.

In one instance, a child, aged 6 years, had the daily habit of getting into a catatonic position late in the afternoon with his body curved forward, his neck backward and his eyes directed upward as if toward some picture high in the air and maintaining this position continuously throughout the night until 7 or 8 o'clock the next morning, when he would go to sleep, only to resume the strange position the following afternoon. This he continued for about two months, when he was first brought for study. There was a definite history of encephalitis with double vision and other clinical concomitants four months preceding the psychosis, which was accompanied by partial speech inhibition, unwillingness to take food and other commonplace symptoms suggestive of catatonic dementia praecox. The spinal fluid contained 8 cells per cubic millimeter, with no other changes, and puncture was followed by temporary recovery with resumption of the catatonic position two months later. Further track of him has been lost. The mother of this child was a psychopathic inferior, and the father a nonentity.

Three other cases of well defined dementia praecox type were noted, each following seemingly indisputable attacks of epidemic encephalitis. In all of these cases there was good reason to believe that the psychosis was that of dementia praecox and that the encephalitis acted as an excitant of predisposed patients, just as war shock or other unusual experience acts on psychopathic personalities.

The most disastrous psychosis occurred in a man, aged 59, who, following a personally observed prolonged typical attack of epidemic encephalitis, returned a year later. At this time he suffered from typical paranoidal delusions of persecution, with hallucinations of sight and hearing. He believed that his wife was conspiring against him and that she was untrue to him. He transferred his life insurance to other members of the family, changed his will, made an unsuccessful attempt to kill his wife by shooting her, and then committeed suicide. Prior to his infection they had lived amicably, but he had always been penurious in proportion to his wealth, peculiar in certain ways, and there was a strong suggestion that the encephalitis acted as an excitant rather than the entire cause of his psychosis.

\section{NEURASTHENIA AND PSYCHASTHENIA}

The largest group (Group 3) was made up of those who became neurasthenic or psychasthenic or both. The exact number so affected is not definitely ascertainable for, on the one hand, they departed from the normal so little and, on the other hand, shaded so gradually into the territory of the psychoses that it would be hard to say where neurasthenic and psychasthenic sequelae began or ended. Almost constant sub- 
normal blood pressure, anemia, vertigo, fatigability, deranged appetite, gaseous eructations, spinal weakness, tachycardia and palpitation, difficulty in mental concentration, poor memory, restlessness and insomnia (especially severe in two cases) form an unmistakable complex. Of interest in connection with the preceding double vision was the persistence of oculomotor asthenia, in some cases still present at the end of three and one-half years since the original infection. These patients uniformly complained of difficulty in reading, typified in the statement of one of them made three years after infection, who said, "I can use my eyes very well, but they do not want to work." With ordinary test cards his vision was good, there was no difficulty in distinguishing words or letters, but after reading a short time he wanted to quit, the type blurred, his eyes were fatigued, his mind would not concentrate, and he soon gave up reading as an unpleasant and laborious task. Though previously a capable electrician, he is still unable to work except part time and on small, unimportant jobs. Most of the patients with this oculomotor fatigue dislike moving pictures, bright lights, undue exposure to light, and any labor which involves prolonged and concentrated thought or action. In a word, they are typical psychoneurasthenics with predominance of oculomotor symptoms. It is hard to escape the conviction that some of them were neurasthenics in advance of the affection. In fact, with several of the worst cases this is known to have been the (ase. In varying degree these neurasthenic symptoms remain in a large percentage of the entire group which forms the basis of this discussion. Many belonging to Group 3 should recover.

Fundus examination in many cases suggests certain changes which, owing to apologetically acknowledged lack of real expertness with the ophthalmoscope, may be imperfectly described, consisting of slight blurring and indistinctness of the outlines of the disks and of the retinal blood vessels, both veins and arteries. There was no case of true papillitis or papilledema or optic nerve atrophy, and such changes as persist are comparatively slight and might escape notice were one not looking for them. Pupillary inequalities and sluggish reactions, especially in the parkinsonian cases, were common, and in at least three cases rigid pinpoint (Argyll Robertson?) pupils were noted.

\section{ORGANIC LESIONS}

Hemiparesis.-Two patients remain hemiparetic. One was distinctly hemiplegic for six months, after which his gait and faulty hand position slowly improved. The leg is still slightly rigid, and the forearm and hand seek a somewhat flexed position across the lower part of the abdomen in a manner suggestive of hemiplegia. The minds of both these patients have lost their flexibility, and they meet with difficulty in creating new thoughts or new ideas, though both pursue their businesses reasonably well but without the vigor and enthusiasm of bygone days.

Pontile Symptoms.-Several patients still exhibit weakness of one or more ocular muscles accompanied by slight strabismus which they are able to overcome temporarily by an effort. One man has a rather complete ophthalmoplegia, with marked limitation of motion of the eyeball in all directions. A woman, one year after an attack of fever accompanied by double vision from which she recovered, suddenly without warning developed inability to raise her eyeballs above the horizontal level, that is, she has paresis of both superior rectus muscles. There is some ptosis, and her habitual attitude is one in which the eyeballs turn down as if she was reading a book in her lap. All of the patients with oculomotor palsies excepting the last one belong to the parkinsonian group, but this one has no other symptoms suggestive of that disorder. These oculomotor symptoms seem clearly to point to a chronic poliencephalitis superior.

Bulbar Symptoms.-Those who present sequelae suggesting lesions of the medulla were relatively few, probably because lesions of the medulla were usually fatal, except as medullary symptoms were present in certain of the parkinsonian cases. Three patients with typical parkinsonian symptoms periodically find difficulty in swallowing, and suffer from excessive salivary secretion and constant trembling about the lips and tongue which suggest incipient bulbar paralysis. Since there has been a steady advance in the symptoms of these over many months, there is justification for the fear that they will eventually die from involvement of the bulb.

Parkinsonian Cases.-Twelve patients with a marked parkinsonian syndrome remain, 10 per cent. of the nonfatal group. At least four of the fatal cases were of the parkinsonian type, so that the total number of cases of this complex was approximately 11 per cent. In addition, some of those classified as neurasthenics showed slight blankness of expression, rigidity of movement and other mild parkinsonian symptoms, which have gradually diminished. The parkinsonian disorder was characterized by the early development of loss of expression, staring, unwinking eyes, muscle rigidity and gait difficulties, all of a type similar to the second stages of paralysis agitans but without the characteristic tremor. Three patients suffer from excessive and annoying salivary secretion. In addition to the more or less characteristic rigidities there was, on occasion, a tendency to the assumption of strange and unusual positions, in a sense not dissimilar from that already described in the child who appeared to suffer from catatonic dementia praecox. A man, aged 23, who is excessively rigid, has lain for many months face downward on a couch with his hands drawn forward under him in characteristic parkinsonian fashion. When erect, he finds difficulty in turning his body, moves slowly, and walks with a rigid gait. When left alone he resumes the prone position. His mind acts slowly but clearly, and his speech is hesitating.

It was early noticed that these patients lacked the characteristic tremor of agitans. Some tremor has been present in most of the cases, but with one exception has never been constant. It has sometimes appeared in one hand or one leg, sometimes has been under control, and sometimes has suggested the intention type; but, in the main, when patients were under observation it has been absent, that is, controlled. An exceptional tremor occurred in a patient already described as suffering from ophthalmoplegia. This man suffers from continuous tremor involving his entire body, including his vocal cords, so that his voice has a bleating sound. The tremor lacks the characteristic pill rolling motion, and is much more rapid than agitans.

Parkinsonian symptoms appeared in patients from 21 to 65 years of age. In only one of this group were the characteristics typical of paralysis agitans. This was in a man, aged 65, who gave a clear-cut history of onset 
with double vision, fever and lethargy, but was unable to tell when he first began to shake. It seems reasonable to suspect that here was a patient with incipient paralysis agitans who also had the misfortune to contract epidemic encephalitis.

A final curious result of complication of the parkinsonian sequelae occurred in a woman, aged 48 , who now, nearly three years after the date of infection with typical epidemic encephalitis, is expressionless, rigid in gait, and has a blood picture of pernicious anemia.

\section{COMBINED AND ATYPICAL SEQUELAE}

Though an attempt has been made to separate the different types of sequelae of epidemic encephalitis, it has not been easy to classify every patient, for the reason that some patients could be classified in two or even three groups. Some of the neurasthenic patients had partial ophthalmoplegic disturbances. A violently agitated patient, twice discussed, suffered from parkinsonian syndrome, ophthalmoplegia and pronounced psychosis and muscular jerking; in fact, he might have been classified in any of the groups.

Occasionally, patients have complained of atypical sequelae, such as neuritic pains which were not of sufficient frequency or distinctness to warrant separate classification. No postencephalitic convulsions occurred. There was apparently complete absence of visceral lesions as distinct sequelae, though in two cases, after the acute onset of the disease, glycosuria of marked degree was observed, and it is possible that search over the entire group might reveal other cases showing visceral lesions. It is probable that in both these cases the glycosuria was due to irritation of the fourth ventricle, but it is not unreasonable to assume that, if long continued, such irritation might result in permanent changes in the glycogen-forming organs.

306 Selling Building.

\section{ABSTRACT OF DISCUSSION}

DR. M. A. Bliss, St. Louis: Among the sequels of epidemic encephalitis as observed in St. Louis, the most striking thing we noted was the great similarity to the remissions and exacerbations of multiple sclerosis. We have patients who improve so markedly that we think they are well on toward recovery, and then the symptoms recur. That has happened in our experience many times.

DR. F. E. Covlter, Omaha: We are very frequently asked: Will these patients recover? How long are they going to be ill? Are they going to be as well as before they were attacked with this disease? Sufficient time has probably not yet elapsed to answer those questions accurately. I was glad to hear the optimistic views expressed by Dr. House. My own experience has not been quite so optimistic, especially with those persons who have had this disease in early life. I wish he would tell us concerning these results in children, The cases in children seem to have resulted in the most havoc, for the greatest destruction to the mental and nervous apparatus is found in the children. The longer the duration, the more severe the condition has been; also, the longer the period of lethargy, the more serious has been the result so far as sequels are concerned, especially in connection with children.

Dr. Walter L. Timme, New York: I believe that the death rate is not nearly so high as Dr. House represents his to have been. I think the reason for the high death rate reported by him is that a great many persons with the disease in a very mild form seldom come under the observation of the specialist or go to the hospital. These cases are seen by no one at all, or by a family practitioner, who sees the patient for a day or two in a mild febrile state, prescribes some therapeutic treatment, and the patient recovers. I am convinced that a great many cases of this kind escape us entirely, and hence our mortality statistics must be taken with that reservation. Dr. House mentioned the Argyll Robertson pupil. I have never seen in these cases a true Argyll Robertson pupil. It is a pupil which reacts perhaps not at all to light, or possibly very sluggishly, but is also accompanied by an accommodation spasm, so that we have a definitely fixed pupil rather than one that reacts to light and not to accommodation. Occasionally we see a case that shows the marked effect of the infection on the vegetative nervous system and the related organs. In a certain number of cases these patients become very adipose as a result of the attack, an increase in weight of 60 or 80 pounds in the course of six months being not at all uncommon. Another type of case seen only seldom is one in. which there is a bronzing of the entire body within four or six months after the attack of encephalitis. The intense asthenia seen in true Addison's disease is also present.

Dr. George A. Moleen, Denver: I do not think any disease has been fraught with so many difficulties particularly directed to the neurologist as epidemic encephalitis. I am frank to confess that that has been my experience, and I have derived a great deal of consolation from the fact that both the French and the German journals are replete with instances of the same kind. The widest divergence in syndromes is evident in these reports, in conformity with my own experience. I was interested in the classification brought out by Walsh, in which he segregated the cases into two groups, in accordance with the positive and negative classification of other conditions by Hughlings Jackson. By the positive cases he referred to those which had active muscular contractions with the active mental symptoms, the nystagmus, and the irritative sensory disturbances, particularly pain. The negative types included the anesthesias and paralyses with the associated atrophies, the somnolence and lethargy, and the pupillary or eye paralyses with them. He stated that the prognosis was universally better in the paralytic types than in those which showed the muscular or mental activities, and that has been my experience. Also in the active motor types the sequels are more likely to occur. At the New Orleans session I related a case which had not terminated at that time. The patient was admitted with a bulbar paralysis, the inability to swallow, and paralysis of the tongue. Atrophy of the tongue with sagging of the palatine pillars was followed by paralysis of the shoulder girdle, and a poliomyelitic type paralysis of the lower extremities. Recovery occurred in the same order, and I remember mentioning that his ability to swallow recurred with the presentation of a dish of strawberries. The man went on to complete recovery, and not a single evidence of his condition remains. This is only one case in conformity with Walsh's contention.

Dr. W. A. Jones, Minneapolis: We see so many cases that resemble encephalitis that I think for a time we were inclined to diagnose almost every neuropsychiatric condition as encephalitis. I distinctly regret that in one case I emphatically decided that a woman who had a few psychiatric symptoms had an encephalitis. She was sent to the hospital, and within twenty-four hours developed a stuporous state that resembled the stupor of a lethargic encephalitis; she wakened for her meals and was conscious of the activities of the nurse. A puncture of the spinal canal revealed that she had a four plus Wassermann reaction. $\mathrm{Her}$ stupor became intensified, and she died in a week. The necropsy revealed nothing in the way of an encephalitis. Aside from her Wassermann reaction she had a large intraventricular tumor of the brain. Previous to seeing this case I had a similar case in which there was the same attitude of somnolence without symptoms of encephalitis, and, singularly, the woman had a large brain tumor. With this confusion of symptoms in the cases that we see now we should be reasonably careful in coming to a definite conclusion.

Dr. Leo M. Crafts, Minneapolis: One thing that has impressed me is the uncertain chronicity of this condition. I do not think that we yet know how long the process may continue, either directly or its sequels. I have in mind a case of encephalitis in which there was a quite typical attack with paralysis of the left face and arm, with lethargy. The patient apparently made a fair recovery, but was not in really good condition. The following winter he presented 
the same clinical picture again and died. I am now observing a young ex-service man who had an attack of influenza in the fall of 1918 and was never well afterward. More than a year later he developed the active picture of epidemic encephalitis. He gradually improved, but has continued to show the parkinsonian picture since that time, without tremor, but with the boardlike expression and automatic movement. $\mathrm{He}$ is now steadily retrograding. I believe that he has a continued encephalitis, either a chronic process without the activity of a germ or with it. The cases that have impressed me most have been those that resembled brain tumor. In one case the condition began as a simple nervous prostration, but gradually retrograded. The patient continually spat, an unusual symptom. Later he developed an active cephalalgia that did not yield at all to treatment. He lost motion on the left side, which symptom disappeared. He developed double choked disk, but this definitely receded after spinal puncture and one drainage, and did not recur. In the light of experience with this case I think that this fact, together with the rapid qualitative change in the reflexes from normal to abnormal and back, are the best differential points between actual brain tumor and encephalitis. In the case referred to, resembling brain tumor, with cerebellar nystagmus and falling backward, a double decompression was done at the occipital level. Microscopic examination of the brain revealed some of the characteristic signs of cell infiltration about the blood vessels, but nothing else. I have seen one case simulating spinal cord tumor, beginning with a definite zone of pain in the lower back and only after several months developing delirium, diplopia and the ordinary progress. One of the many striking things is the almost unfailing marked elevation of temperature toward dissolution, usually going to $106 \mathrm{~F}$. or above.

Dr. Julius Grinker, Chicago: The question of tumor that may be diagnosed when there is an encephalitis, and, on the other hand, the diagnosis of encephalitis when there is a tumor, was strikingly brought out by a case of encephalitis with choked disk. A colleague had a case with choked disk, which he presented as a case of encephalitis, characterizing it as the second case of encephalitis with choked disk, mine being the first-choked disk being a very unusual symptom of epidemic encephalitis. My colleague's patient died, and the condition proved to be a true tumor of the brain. My patient exhibited choked disk and also showed symptoms which Dr. Crafts referred to and form which he would have made a diagnosis of tumor, but the patient made a perfect recovery. His was a true case of encephalitis. This goes to show that we are likely to mistake organic disease of other types for encephalitis, and vice versa. An interesting point is this: Recently I saw a number of sequels of encephalitis in cases which were never diagnosed as encephalitis at all. These sequels were mostly in the form of postencephalitic rigidity and parkinsonian tremors. I have seen many cases which exhibited this syndrome. These cases occur with preference in young persons. A young boy had what was undoubtedly an encephalitis lasting for six months, during which he was apathetic, drowsy and in bed most of the timethe case was not diagnosed as encephalitis. The condition began with ocular phenomena, a little fever, and undoubtedly must have been . encephalitis, though I had no opportunity of seeing the case myself at that time. This boy now presents the following complications, which some one called a genuine sequel of encephalitis: Every alternate morning, sometimes every morning, the boy, after awaking, would run about the room in a frenzy, swear at his mother and jump at her. Aside from these attacks, he was a very well behaved child. This sequel was directly attributed to the encephalitis. The diagnosis appeared doubtful to me. The mother described his attacks as being accompanied by blanching at the lips with change in color of the face, and the suggestion arose: May this not be a form of epilepsy? I gave him phenobarbital (luminal) and he did not improve. Finally, after studying the case $I$ found that at times he did not swear, especially when something harsh was said to him. I then advised the mother to spank the boy every time he had an attack, and he has not had any more. The case was evidently a neurosis of the hysteria group.

Dr. Charles R. Ball, St. Paul: I remember the first time I saw one of those parkinsonian types of encephalitis. It was at Base Hospital 9, at Chateroux, in 1918. I was called in consultation to see a young soldier who was sitting up in bed with a perfectly masklike expression on his face, and a marked degree of muscular rigidity. I was told that he had recently had a rather severe attack of influenza in which he had been delirious for a number of days. I recognized immediately that here was something new in my experience, something entirely different than I had ever seen before. A few weeks later at Base Hospital 214 at Savaney, at one of our weekly clinical conferences, we were able to show seven cases of this type of encephalitis. The literature has since been filled with a protean symptomatology of the various types of this disease, which present definite somatic findings. At present, because of this extensive literature on this subject, when there are somatic symptoms, the recognition of the nervots sequelae of this disease is not difficult. But what about the other sequels, when there are no objective symptoms, when the history of the attack is vague, and when we have to deal simply with changes in the personality, or with symptoms entirely referable to the vegetative nervous system? We are called on in such cases to explain strange and unfamiliar nervous and mental conditions, which we have previously classified under hysteria and psychoneuroses. We see undoubtedly many such cases among ex-service men, that at present, because of our lack of understanding of these sequels, we are classifying as shell shock. It is in this type of postencephalitic sequel that our most difficult problems in nervous diagnosis are to be found at present.

Dr. J. C. Michael, Minneapolis: A case which interested me very much was that of a young man who had delirium for about three weeks. He became quite markedly parkinsonian for a year and a half. Then he suddenly developed acute symptoms again and died. At necropsy the usual pathologic changes of epidemic encephalitis were presented.

Dr. Frank A. Ely, Des Moines: I wish to report one case treated by thyroid. A girl, aged 17 , became ill one year before I saw her. She was a pitiable object, unable to continue her work in high school, a typical parkinsonian endresult. As an empiric treatment $I$ asked her physician to give her from 3 to 5 minims of 1: 1,000 epinephrin solution hypodermically every other day, and to administer small doses of thyroid, beginning with 1 grain a day and increasing the dose to 5 grains. Within sixty days this girl had regained her usual alert state; her mentality improved; she was able to go about and resume her studies, and recently, about six months after institution of the treatment, I received a report that she was apparently perfectly well. I realize that one case means nothing: it is simply a report for the sake of stimulating observations along that line. I have had one other typical parkinsonian case in a service man who had peculiar respiratory phenomena in which he snorted all the time, a peculiar forcible exhalation. He was very much improved as long as we kept him on the thyroid and epinephrin. As soon as the treatment was discontinued, his respiratory difficulty would return.

Dr. William House, Portland, Ore.: Dr. Bliss refers to multiple sclerosis cases. Dr. Bassoe first called attention to the hope that in encephalitis we might find the etiology of paralysis agitans and multiple sclerosis. I think that his hope has not been fulfilled. The results of encephalitis do not appear to be typical of agitans or multiple sclerosis. Dr. Coulter speaks of my optimism in prognosis. I pointed out the difference that existed in opinions, and think my prognoses not too optimistic. I believe that the last group that I presented, Group 4, will have no recoveries, but that the neurasthenic and myoclonus cases will recover. I saw very few children. Most of the cases of encephalitis in children that I saw were fatal. Answering Dr. Timme's comment on the high death rate, six of my first thirteen cases were fatal, but as the epidemic advanced the cases seemed less virulent, in this respect corresponding to experiences with poliomyelitis in which the first epidemic was always much more severe than the recurrences of the following winter. In the second series of cases of encephalitis there were many of the mild type described by Dr. Timme, patients with a temperature of about 101 or $102 \mathrm{~F}$, some delirium and a few days of double vision followed by lethargy and recovery. In regard to the Argyll Robertson pupil, I think that Dr. Timme made a just 
correction. These pupils are not Argyll Robertson; they are simply contracted pupils in which responses to both light and distance are greatly diminished. Dr. Moleen speaks of nystagmus. It seems to me that there is no true nystagmus in these cases. The wavering motions that exist are due to muscle weakness, and are a pseudonystagmus and not a true one. As to prognosis in paralytic cases: Mine were severe cases. The patients have not done well, and all of them have some sequel. Of the myoclonus patients, some have died and others have severe mental and physical sequels, except the two mentioned. In regard to the difficulties in diagnosis, I encephalitis, especially tuberculous meningitis and encephalitis. In most cases it should be fairly easy to make an accurate diagnosis. A final suggestion in connection with the association between encephalitis and various psychoses may be of interest. Most of my patients were in poor health when they developed encephalitis. They were subnormal, and, in view of my belief that the psychotic cases occurred in psychopaths, it seems to me that subnormal persons are more predisposed to the disease than normal persons.

\section{Clinical Notes, Suggestions, and New Instruments}

\author{
DEAFNESS FROM PRESENCE OF INSECT IN EAR
} found some trouble in differentiating between brain tumor and

added information in suspected lesions of the stomach and duodenum. The entire procedure is as follows:

The patient abstains from meat on the day the test is done, or for a longer preceding interval if symptoms of pyloric obstruction are present. A "pearl" button (from 7 to $8 \mathrm{~mm}$. in its greatest width) attached to a thread of braided silk is swallowed by the patient at 9 p. m. and washed down by a glass or two of water. A knot at $75 \mathrm{~cm}$. marks the limit to which the thread is allowed to travel. The next morning a mark is made at the part in contact with the teeth, and the thread and button are removed before any food has been given. Successive strips of $10 \mathrm{~cm}$. each, starting from the external dental mark, are tested in a small porcelain dish with the benzidin and hydrogen peroxid mixture which is commonly used for occult blood tests. Stains due to blood assume a green color, and the proximal stain, counting from the mouth end, may be taken as the probable distance of a bleeding area. However, it is not advisable to regard the calculation of this possible site as being positively established. A sensitive benzidin is of value, and a hydrogen peroxid solution not containing acetanilid is preferable. It is possible to make a preliminary trial, using a two or three hour interval only; in some cases this is sufficient to give enough blood impregnation to react definitely. In case of doubt, however, the longer time given above is the method of choice. Oxidations of the benzidin due to the presence of oxydases have not been noted in this technic.

2035 Chestnut Street.

\section{A NEW SEPTAL CHISEL}

A man, aged 36, stumbled and fell, Feb. 18, 1922, striking on his chin. He was unconscious for a few seconds and, on recovering, experienced a sharp pain in his right ear. After the chin wound was dressed he was referred to me for examination of the ear. The external canal was blocked. A large amount of débris was removed, disclosing tightly packed cerumen, which was removed with difficulty. Following this came the remains of a dead cockroach, $18 \mathrm{~mm}$. in length and perfectly preserved in every detail.

The patient in April, 1908, while preparing for a bath, felt a sudden pain and buzzing in the right ear, which drove him almost frantic. While his wife was telephoning for a physician, he put his head under the water in the tub, and the pain soon ceased. Later, on the advice of his physician, the ear was syringed, but nothing was washed out of the ear, and it was supposed that the trouble was due to a small mite which had been lost in the washing. The patient gradually became deaf in this ear, but never consulted a physician, believing that some irreparable damage had been done to the ear by the insect. After removal of the insect and cleansing of the canal, hearing was fully normal, and the only evidence of injury was a slight abrasion of the drum.

It seems probable that, following the original injury in 1908, the canal became gradually filled with wax, thus entombing the insect in an air-tight space and preserving it in toto for nearly fourteen years.

221 Meyers Arcade.

\section{MODIFIED EINHORN THREAD TEST}

\section{Edwakd Steinfield, M.D., Philadelphia}

The equivocal results in the use of Einhorn's thread test in the diagnosis of gastric ulcer and malignancy have been due in part to the difficulty in the recognition of the stains as blood. Though other objections have been advanced from time to time by various observers, it has appeared that modifications tending to render the test more sensitive would be of material aid in increasing the usefulness of the procedure. The outlining, by chemical methods, of the areas on the thread impregnated with blood, renders the identification of faint or doubtful blood stains less difficult. A method utilizing this principle has been used recently, and the results are sufficiently encouraging to warrant its trial as a source of

\section{Creighton Barker, M.D., New Haven, Conn,}

This instrument is designed for the removal of the base of the nasal septum and septal ridges. It has been used by a number of rhinologists with gratifying results. Essentially, the instrument is a Freer chisel to which tiny rounded knobs have been added to protect the sharp corners of the blade. The advantage of this protection are clear-it is practically impossible to injure with it the overlying mucous membrane. The projections at the sides of the blades also make it easier

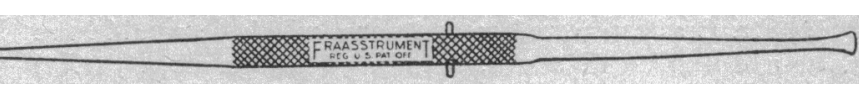

Septal chisel.

to hold the cutting edge against a narrow projection without slipping, and its use with the blade vertical, as in severing a spur, is facilitated thereby.

66 Trumbull Street.

\section{A FILTERJNG ADAPTER FOR THE ADMINISTRATION OF NEO-ARSPHENAMIN}

Jay F. Schamberg, M.D., Philadelphia

During the World War, the practice became common abroad of administering neo-arsphenamin in concentrated solutions in a small Luer syringe. This practice has likewise been adopted by many physicians in this country. It possesses the advantage of simplicity of administration and of time economy, factors which are important when large numbers of patients in clinics are treated.

When concentrated solutions are used with a Luer syringe, and even when larger quantities of water are employed, it is quite possible for minute fragments of glass dust, dust in the water or undissolved particles of the drug to be carried through the needle. Such particles, while not large enough to be readily visible to the eye, may still be of sufficient size to cause an embolism in the minute capillaries of the lungs or possibly other organs. It is probable that the nitritoid and similar immediate reactions which follow the administrations of arsphenamin are due to embolic processes. It is now common knowledge that solutions of neo-arsphenamin

\footnotetext{
* From the Ear, Nose and Throat Service, Grace Hospital.
} 